\title{
The Effects of Piezoelectricity on the Interaction of Waves in Fluid-Loaded Poroelastic Half-Space
}

\author{
Vishakha Gupta ${ }^{1}$ and Anil K. Vashishth ${ }^{2}$ \\ ${ }^{1}$ Department of Mathematics, Dyal Singh College, Karnal 132001, India \\ ${ }^{2}$ Department of Mathematics, Kurukshetra University, Kurukshetra 36119, India \\ Correspondence should be addressed to Vishakha Gupta; vi_shu85@yahoo.co.in
}

Received 21 October 2013; Revised 9 February 2014; Accepted 6 March 2014; Published 17 April 2014

Academic Editor: Manas C. Ray

Copyright (C) 2014 V. Gupta and A. K. Vashishth. This is an open access article distributed under the Creative Commons Attribution License, which permits unrestricted use, distribution, and reproduction in any medium, provided the original work is properly cited.

\begin{abstract}
The effects of piezoelectricity on the interaction of waves at fluid-poroelastic interface are studied. The constitutive equations and governing equations are formulated and their solution is obtained. The boundary conditions are described at fluid-solid interface. The effects of various parameters on the angle of refraction, amplitude ratios, displacements, electric potentials, and vertical component of slowness are studied numerically for a particular model. The results obtained are in agreement with the general laws of physics.
\end{abstract}

\section{Introduction}

It is well known that piezoelectric materials produce an electric field when deformed and undergo deformation when subjected to an electric field. Piezoelectric materials are acting as very important functional components in sonar projectors, fluid monitors, pulse generators, and surface acoustic wave devices. The field of piezoelectric materials has advanced rapidly due to an increasing awareness about capabilities of such materials, the development of new materials and transducer designs, and increasingly stringent design and control specifications in aerospace, aeronautics, industrial, automotive, biomedical, and nanosystems. Engineering applications in surface acoustic waves (SAW) devices, materials characterizations, and smart structures require the analysis of elastic wave interaction with piezoelectric materials. The problem of reflection-transmission in piezoelectric materials has been attracting unceasing attention in view of its theoretical interest and from application points of view also. The characteristics of the reflected and refracted waves at such boundaries give information about the resolution characteristics of acoustic transducers [1]. The reflection and refraction of plane waves in piezoelectric anisotropic materials have been mentioned in the texts of Auld [2], Dieulesaint and Royer [3], and Parton and Kudryavtsev [4].
Noorbehesht and Wade [5] obtained the analytical expressions for reflection and transmission coefficients of the waves at a boundary between piezoelectric materials and water and studied the effects of angle of incidence and material properties on these coefficients. Auld [6] studied the wave propagation in piezoelectric materials. The reflection of a transverse wave from the surface of a piezoelectric crystal of class 6 was studied by Alshits et al. [7]. Nayfeh and Chien [8] made an analytical study on ultrasonic wave interaction with fluid-loaded anisotropic piezoelectric substrates. The analytical expressions for the reflection and transmission coefficients were derived and propagation characteristics of the leaky wave and free wave are also identified. Every and Neiman [9] analyzed the reflection of electroacoustic waves at the boundary of a piezoelectric half-space. Zinchuk and Podlipenets $[10,11]$ obtained dispersion equations for acoustoelectric Rayleigh wave in a periodic layer piezoelectric half-space in a study for the $6 \mathrm{~mm}$ crystal class. Zaitsev and Kuznetsova [12] performed a detailed analysis of energy characteristics of bulk, surface, and plane acoustic waves in piezoelectric materials and structures. Le Clezio and Shuvalov [13] dealt theoretically and experimentally with the transmission of acoustic waves through a piezoelectric plate. Burkov et al. [14, 15] derived the basic equations describing the wave at the interface between acentric crystals 
and studied the effects of a uniform external electric field on the reflection and transmission of waves. The role of electromagnetic effects in the reflection and transmission of acoustic waves in piezoelectric materials was discussed by Darinskii et al. [16]. Abd-Alla and Alsheikh [17] studied the reflection-transmission of quasi-longitudinal waves under initial stresses at an interface of two anisotropic piezoelectric media with different properties.

Porous piezoelectric materials are widely used for applications such as low frequency hydrophones, miniature, accelerometers, vibratory sensors, and contact microphones. Different theoretical models [18-21] were developed to study the effects of porosity and connectivity on the elastic, dielectric, and piezoelectric coefficients of piezoelectric composites. Different experimental studies [22-25] were made on manufacturing of porous microstructures and the properties of such porous piezoelectric materials. Vashishth and Gupta $[26,27]$ derived the constitutive equations for porous piezoelectric materials and studied the wave propagation in these materials. The general theorems of elasticity were generalized for the linear theory of porous piezoelectric materials by Vashishth and Gupta [28]. Recently, Vashishth and Gupta [29] studied the effects of piezoelectricity on the poroelastic plate sandwiched between fluid half-space and porous piezoelectric half-space. The effects of angle of incidence and frequency on the amplitude ratios are studied therein.
Survey of the literature related to porous piezoelectric materials reveals that a lot of experimental work has been done and is being done. Very few authors have established theoretical models for porous piezoelectric materials. A lot of work has also been done on reflection and transmission of waves in piezoelectric materials but theoretical work in porous piezoelectric materials is comparatively less reported and thus finds scopes for further investigations. In the present paper, the effects of piezoelectricity on the interaction of waves in fluid-loaded poroelastic half-space are studied. In the present model the porous piezoelectric plate is sandwiched between two fluid half-spaces. The formal expressions for the mechanical displacements, electric potentials, stresses, and electric displacements are obtained for the porous piezoelectric half-space. The effects of various parameters on the amplitude ratios, displacements, electric potentials, angle of refraction, and vertical component of slowness are also studied numerically for a particular model.

\section{Geometry of the Problem}

Let us consider a poro-piezoelectric half-space loaded with fluid half-space. Let a plane wave from the fluid half-space strike the interface, which results in one reflected wave and five transmitted waves in poro-piezoelectric half-space.

The constitutive equations for porous piezoelectric materials, having $6 \mathrm{~mm}$ symmetry, are [26]

$$
\left[\begin{array}{l}
\sigma_{11} \\
\sigma_{22} \\
\sigma_{33} \\
\sigma_{23} \\
\sigma_{13} \\
\sigma_{12} \\
\sigma^{*} \\
D_{1} \\
D_{2} \\
D_{3} \\
D_{1}^{*} \\
D_{2}^{*} \\
D_{3}^{*}
\end{array}\right]=\left[\begin{array}{cccccc}
c_{11} & c_{12} & c_{13} & 0 & 0 & 0 \\
c_{12} & c_{11} & c_{13} & 0 & 0 & 0 \\
c_{13} & c_{13} & c_{33} & 0 & 0 & 0 \\
0 & 0 & 0 & c_{44} & 0 & 0 \\
0 & 0 & 0 & 0 & c_{44} & 0 \\
0 & 0 & 0 & 0 & 0 & \frac{\left(c_{11}-c_{12}\right)}{2} \\
m_{11} & m_{11} & m_{33} & 0 & 0 & 0 \\
0 & 0 & 0 & 0 & e_{15} & 0 \\
0 & 0 & 0 & e_{15} & 0 & 0 \\
e_{31} & e_{31} & e_{33} & 0 & 0 & 0 \\
0 & 0 & 0 & 0 & \zeta_{15} & 0 \\
0 & 0 & 0 & \zeta_{15} & 0 & 0 \\
\zeta_{31} & \zeta_{31} & \zeta_{33} & 0 & 0 & 0
\end{array}\right.
$$

Here, $c_{i j}$ are the elastic stiffness tensor components of porous bulk material. $R$ is elastic constant corresponding to fluid phase. $e_{31}, e_{33}, e_{15}, \zeta_{31}, \zeta_{33}, \zeta_{15}, e_{3}^{*}, \widetilde{\zeta}_{3}$ are piezoelectric coefficients. $\xi_{11}, \xi_{33}, A_{11}, A_{33}, \xi_{11}^{*}, \xi_{33}^{*}$ are the dielectric coefficients. $m_{11}, m_{33}$ are elastic coupling coefficients. $\sigma_{i j}, \varepsilon_{i j}, D_{i}$, and $E_{i}$ are components of the stress tensor, strain tensor, electric displacement vector, and electric field vector for the solid phase of the porous aggregate, respectively. $\sigma^{*}, \varepsilon^{*}, D_{i}^{*}$, and $E_{i}^{*}$ are the stress, strain, electric displacement components, and electric field components corresponding to the fluid phase of the aggregate.

$\left.\begin{array}{ccccccc}m_{11} & 0 & 0 & -e_{31} & 0 & 0 & -\zeta_{31} \\ m_{11} & 0 & 0 & -e_{31} & 0 & 0 & -\zeta_{31} \\ m_{33} & 0 & 0 & -e_{33} & 0 & 0 & -\zeta_{33} \\ 0 & 0 & -e_{15} & 0 & 0 & -\zeta_{15} & 0 \\ 0 & -e_{15} & 0 & 0 & -\zeta_{15} & 0 & 0 \\ 0 & 0 & 0 & 0 & 0 & 0 & 0 \\ R & 0 & 0 & -\widetilde{\zeta}_{3} & 0 & 0 & -e_{3}^{*} \\ 0 & \xi_{11} & 0 & 0 & A_{11} & 0 & 0 \\ 0 & 0 & \xi_{11} & 0 & 0 & A_{11} & 0 \\ \widetilde{\zeta}_{3} & 0 & 0 & \xi_{33} & 0 & 0 & A_{33} \\ 0 & A_{11} & 0 & 0 & \xi_{11}^{*} & 0 & 0 \\ 0 & 0 & A_{11} & 0 & 0 & \xi_{11}^{*} & 0 \\ e_{3}^{*} & 0 & 0 & A_{33} & 0 & 0 & \xi_{33}^{*}\end{array}\right]\left[\begin{array}{c}\varepsilon_{11} \\ \varepsilon_{22} \\ \varepsilon_{33} \\ 2 \varepsilon_{23} \\ 2 \varepsilon_{13} \\ 2 \varepsilon_{12} \\ \varepsilon^{*} \\ E_{1} \\ E_{2} \\ E_{3} \\ E_{1}^{*} \\ E_{2}^{*} \\ E_{3}^{*}\end{array}\right]$.

The equations of motion are

$$
\begin{gathered}
\sigma_{i j, j}=\rho_{11} \ddot{u}_{j}+\rho_{12} \ddot{U}_{j}^{*}, \\
\sigma_{, i}^{*}=\rho_{12} \ddot{u}_{j}+\rho_{22} \ddot{U}_{j}^{*}, \\
D_{i, i}=0, \\
D_{i, i}^{*}=0,
\end{gathered}
$$

$(i, j=1,2,3)$. 
Here, $\rho_{11}, \rho_{12}$, and $\rho_{22}$ are the dynamical coefficients. $u_{i}$ and $U_{i}^{*}$ are the mechanical displacement components corresponding to solid and fluid phase.

Further,

$$
\begin{gathered}
\varepsilon_{i j}=\frac{1}{2}\left(u_{i, j}+u_{j, i}\right), \quad \quad \varepsilon^{*}=U_{k, k}^{*}, \\
E_{i}=-\psi_{, i}, \quad E_{i}^{*}=-\psi_{, i}^{*},
\end{gathered}
$$

where $\psi$ and $\psi^{*}$ are electric potential functions for solid and fluid phase of the porous aggregate.

Let us assume that

$$
\begin{gathered}
u_{j}=A_{j} \exp \left(\imath \omega\left(p_{x} x_{1}+p_{z} x_{3}-t\right)\right), \\
U_{j}^{*}=H_{j} \exp \left(\imath \omega\left(p_{x} x_{1}+p_{z} x_{3}-t\right)\right), \\
\psi=\Theta \exp \left(\imath \omega\left(p_{x} x_{1}+p_{z} x_{3}-t\right)\right), \\
\psi^{*}=\Omega \exp \left(\imath \omega\left(p_{x} x_{1}+p_{z} x_{3}-t\right)\right),
\end{gathered}
$$

where $p_{x}$ and $p_{z}$ are the horizontal and vertical components of the slowness vector. $\omega$ is the circular frequency of waves. $A_{j}, H_{j}, \Theta$, and $\Omega$ are the associated amplitudes.

Equations (1)-(4) imply

$$
\mathbf{W Z}=\mathbf{0}, \quad \mathbf{Z}=\left(\mathrm{A}_{1}, \mathrm{~A}_{3}, \mathrm{H}_{1}, \mathrm{H}_{3}, \Theta, \Omega\right)^{T} .
$$

The system (5) has a nontrivial solution if

$$
\operatorname{det}(\mathbf{W})=0 \text {. }
$$

This leads to

$$
c_{1} p^{\prime 5}+c_{2} p^{\prime 4}+c_{3} p^{\prime 3}+c_{4} p^{\prime 2}+c_{5} p^{\prime}+c_{6}=0
$$

where $p^{\prime}=p_{z}^{2}$ and the coefficients $c_{i}(i=1,2, \ldots, 6)$ are given in the Appendix.

The five values of $p^{\prime}$ correspond to the slowness of five coupled waves termed as quasi $P_{1}$, quasi $S_{1}$, and quasi $P_{2}$ and another two waves due to piezoelectric interaction, propagating in a medium. The waves termed as quasi $P_{1}$, quasi $S_{1}$, and quasi $P_{2}$ will be denoted by $q P_{1}, q S_{1}, q P_{2}$, respectively. The mechanical displacements and electric potentials in the poro-piezoelectric half-space can be written as

$$
\begin{aligned}
& \left(u_{1}, u_{3}, U_{1}^{*}, U_{3}^{*}, \psi, \psi^{*}\right) \\
& \quad=\sum_{i=1}^{5}\left(1, r_{1 i}, r_{2 i}, r_{3 i}, r_{4 i}, r_{5 i}\right) \mathrm{Z}_{1 i} e^{\imath \omega\left(p_{x} x_{1}+p_{z} x_{3}-t\right)} .
\end{aligned}
$$

Using (1) and (8), we obtain

$$
\begin{aligned}
& \left(\sigma_{31}, \sigma_{33}, \sigma^{*}, D_{3}, D_{3}^{*}\right) \\
& \quad=\sum_{i} \imath \omega\left(g_{1 i}, g_{2 i}, g_{3 i}, g_{4 i}, g_{5 i}\right) \mathrm{Z}_{1 i} e^{\imath \omega\left(p_{x} x_{1}+p_{z} x_{3}-t\right)},
\end{aligned}
$$

where

$$
\begin{gathered}
r_{1 i}=\frac{\mathrm{Z}_{2 i}}{\mathrm{Z}_{1 i}}, \quad r_{2 i}=\frac{\mathrm{Z}_{3 i}}{\mathrm{Z}_{1 i}}, \quad r_{3 i}=\frac{\mathrm{Z}_{4 i}}{\mathrm{Z}_{1 i}}, \\
r_{4 i}=\frac{\mathrm{Z}_{5 i}}{\mathrm{Z}_{1 i}}, \quad r_{5 i}=\frac{\mathrm{Z}_{6 i}}{\mathrm{Z}_{1 i}} \\
g_{1 i}=c_{55} p_{i}^{\prime}+\left(c_{55} r_{1 i}+e_{15} r_{4 i}+\zeta_{15} r_{5 i}\right) p_{x} \\
g_{2 i}=\left(c_{33} r_{1 i}+m_{33} r_{3 i}+e_{33} r_{4 i}+\zeta_{33} r_{5 i}\right) p_{i}^{\prime}+\left(c_{31}+m_{33} r_{2 i}\right) p_{x} \\
g_{3 i}=\left(m_{33} r_{1 i}+R r_{3 i}+\widetilde{\zeta}_{3} r_{4 i}+e_{3}^{*} r_{5 i}\right) p_{i}^{\prime}+\left(m_{11}+R r_{2 i}\right) p_{x}, \\
g_{4 i}=\left(e_{33} r_{1 i}+\widetilde{\zeta}_{3} r_{3 i}-\xi_{33} r_{4 i}-a_{33} r_{5 i}\right) p_{i}^{\prime}+\left(e_{31}+\widetilde{\zeta}_{3} r_{2 i}\right) p_{x} \\
g_{5 i}=\left(\zeta_{33} r_{1 i}+e_{3}^{*} r_{3 i}-a_{33} r_{4 i}-\xi_{33}^{*} r_{5 i}\right) p_{i}^{\prime}+\left(\zeta_{31}+e_{3}^{*} r_{2 i}\right) p_{x} .
\end{gathered}
$$

$\left(Z_{1 i}, Z_{2 i}, Z_{3 i}, Z_{4 i}, Z_{5 i}, Z_{6 i}\right)$ is the eigenvector of the system (5) corresponding to the $i$ th value of $p^{\prime}$.

The displacements $\left(u_{1}^{f}, u_{3}^{f}\right)$ and normal stress $\left(\sigma_{33}^{f}\right)$ in fluid half-space are

$$
\begin{aligned}
& \left(u_{1}^{f}, u_{3}^{f}, \sigma_{33}^{f}\right) \\
& \quad=\sum_{p=1,2}\left(1, W_{p}^{f}, \frac{\imath \omega \rho_{f}^{u}}{p_{x}}\right) U_{p}^{f} e^{\imath \omega\left(p_{x} x_{1}+(-1)^{p+1} q_{f} x_{3}-t\right)},
\end{aligned}
$$

where $W_{1}^{f}=p_{x} c$ and $W_{2}^{f}=-p_{x} c \cdot \rho_{f}^{u}$ is the upper fluid density. $q_{f}$ is the vertical slowness of wave propagating in the fluid half-space.

The boundary conditions on the interface $x_{3}=0$ are

$$
\begin{gathered}
D_{3}=D_{3}^{*}=\sigma_{13}=0, \quad \sigma_{33}=(1-f) \sigma_{33}^{f}, \\
\sigma^{*}=f \sigma_{33}^{f}, \quad(1-f) \dot{u}_{3}+f \dot{U}_{3}^{*}=\dot{u}_{3}^{f} .
\end{gathered}
$$

Making use of (8)-(12), a nonhomogeneous system of order 6 is obtained. Solution of this system gives the reflected and transmitted amplitude ratios.

\section{Numerical Observations}

In this section, the vertical component of slowness, reflected and transmitted amplitude ratios, mechanical displacements, and electric potentials are calculated numerically for a particular model and their variation with different parameters is observed. Following Kar-Gupta and Venkatesh [30], the elastic, piezoelectric, and dielectric constants for PZT are given in Table 1.

Figure 1 shows the variation of angle of refraction with the angle of incidence. The refraction angles corresponding to $q P_{1}, q S_{1}$, and $q P_{2}$ modes are denoted by $\gamma_{1}, \gamma_{2}$, and $\gamma_{3}$, respectively. The critical angles corresponding to $q P_{1}, q S_{1}$, and $q P_{2}$ modes are $\theta_{1}=13^{\circ}, \theta_{2}=31^{\circ}$, and $\theta_{3}=41^{\circ}$. Thus $q P_{1}$ wave becomes evanescent mode after critical angle $\theta_{1}=13^{\circ}$ and $q S_{1}$ wave does not propagate into the medium after $\theta_{2}=31^{\circ} . q P_{2}$ wave also becomes evanescent mode after 


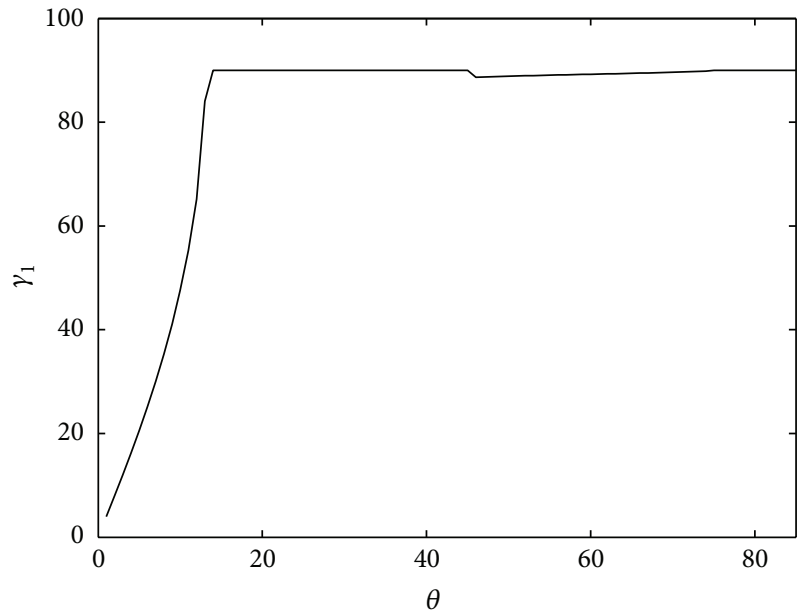

(a)

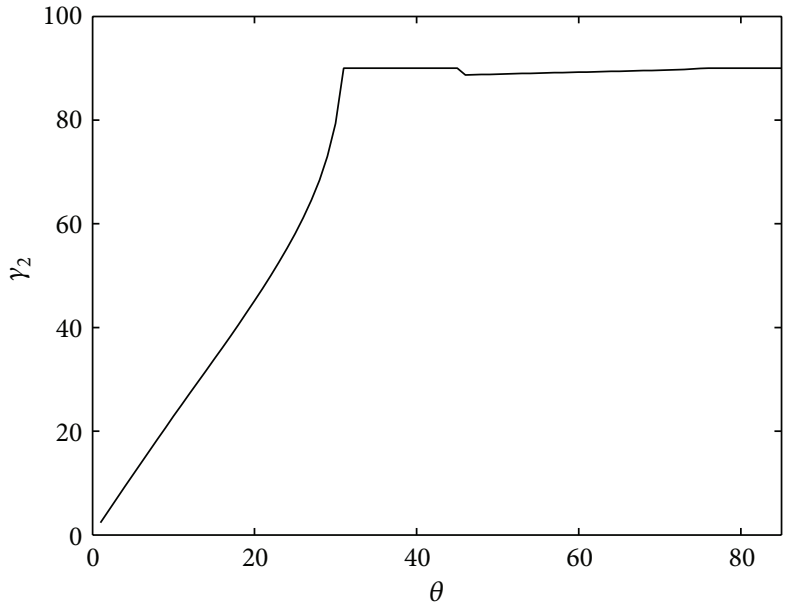

(b)

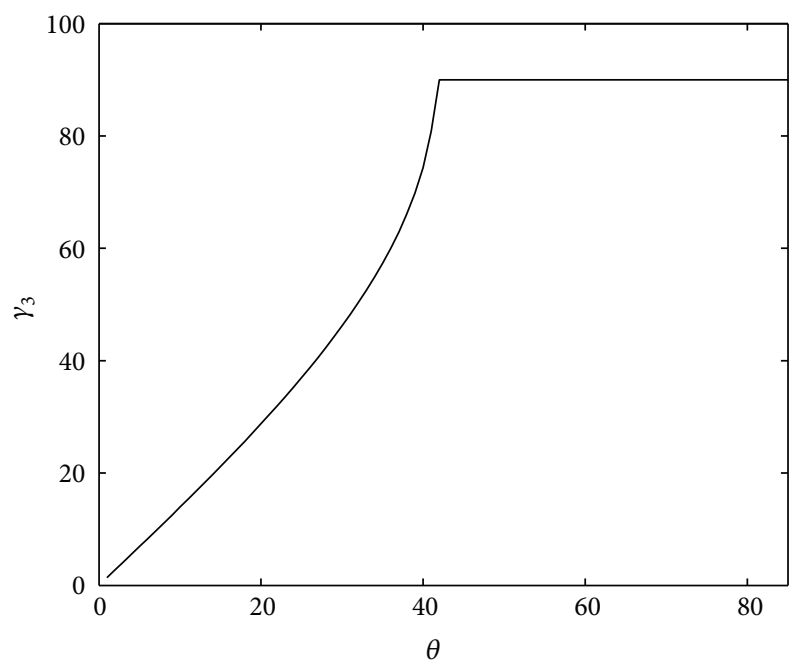

(c)

FIGURE 1: Variation of angle of refraction $\left(\gamma_{i}\right)$ corresponding to propagating modes with the angle of incidence $(\theta)$; (a) $q P_{1}$ wave, (b) $q S_{1}$ wave, and (c) $q P_{2}$ wave.

TABLE 1: Elastic constants, piezoelectric constants, dielectric constants, and other parameters for PZT crystal.

\begin{tabular}{lccc}
\hline $\begin{array}{l}\text { Elastic constants } \\
(\mathrm{GPa})\end{array}$ & $\begin{array}{c}\text { Piezoelectric constants } \\
\left(\mathrm{C} / \mathrm{m}^{2}\right)\end{array}$ & $\begin{array}{c}\text { Dielectric constants } \\
(\mathrm{nC} / \mathrm{Vm})\end{array}$ & Other parameters \\
\hline$c_{11}=148.0, c_{12}=76.2$, & $e_{15}=9.3, e_{31}=-2.324$, & $\xi_{11}=3.984, \xi_{33}=2.081$, & $f=0.2, c_{f}(\mathrm{~m} / \mathrm{s})=1500$, \\
$c_{13}=74.2, c_{33}=131.0$, & $e_{33}=10.99, \zeta_{15}=2.72$, & $\xi_{11}^{*}=11.8, \xi_{33}^{*}=13.9$, & $\left.\mathrm{kg}^{3}\right)=7700$, \\
$c_{44}=25.3, m_{11}=8.8$, & $\zeta_{31}=-0.48, \mathrm{~h}_{33}=5.32$, & $A_{11}=12.8, A_{33}=15.1$. & $\rho_{11}\left(\mathrm{~kg} / \mathrm{m}^{3}\right)=5082$, \\
$m_{33}=5.2, R=20$. & $e_{3}^{*}=-3.6, \widetilde{\zeta}_{3}=-7.5$. & $\rho_{12}\left(\mathrm{~kg} / \mathrm{m}^{3}\right)=-1155$, \\
& & & $\rho_{22}\left(\mathrm{~kg} / \mathrm{m}^{3}\right)=4928$. \\
\hline
\end{tabular}

critical angle $\theta_{3}=41^{\circ}$. All the incident energy gets reflected back after angle of incidence $\theta_{3}=41^{\circ}$.

Figure 2 shows the variation of absolute value of reflected and transmitted amplitude ratios with the horizontal component of slowness. $A_{R}$ corresponds to reflected amplitude ratio and $A_{i}(i=1,2, \ldots, 5)$ denote the amplitude ratios corresponding to transmitted modes. Major portion of incident energy gets reflected back which signifies the fact that the transmitting medium is much denser. The dips in the reflected amplitude ratio correspond to the excitation of pseudosurface mode. Thus we can find the values of incidence angles at which surface modes will be generated. This type of information is of practical importance for design of piezoelectric transducers. The variation of vertical component of slowness with the horizontal component of slowness is shown in Figure 3. The vertical component of 



FIGURE 2: Variation of absolute value of amplitude ratios with the horizontal component of slowness.

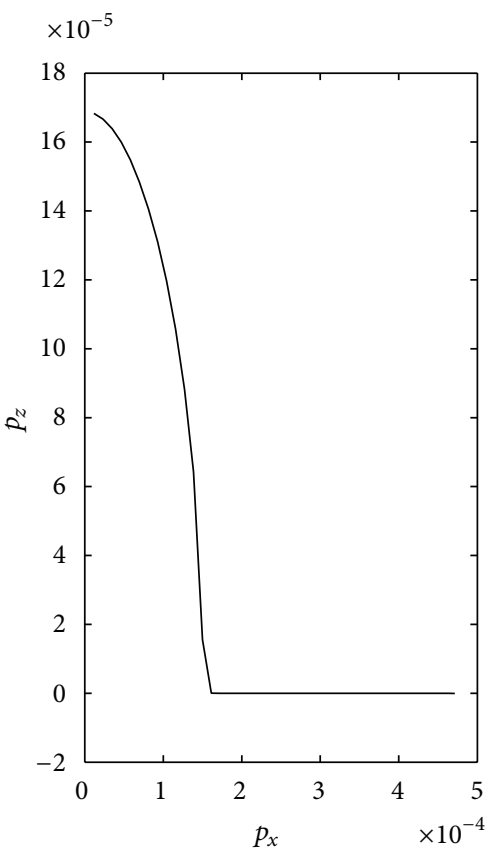

(a)

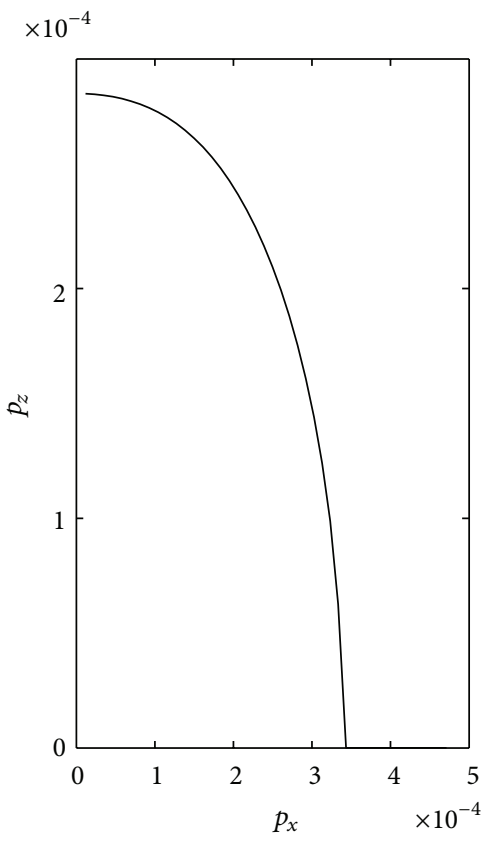

(b)

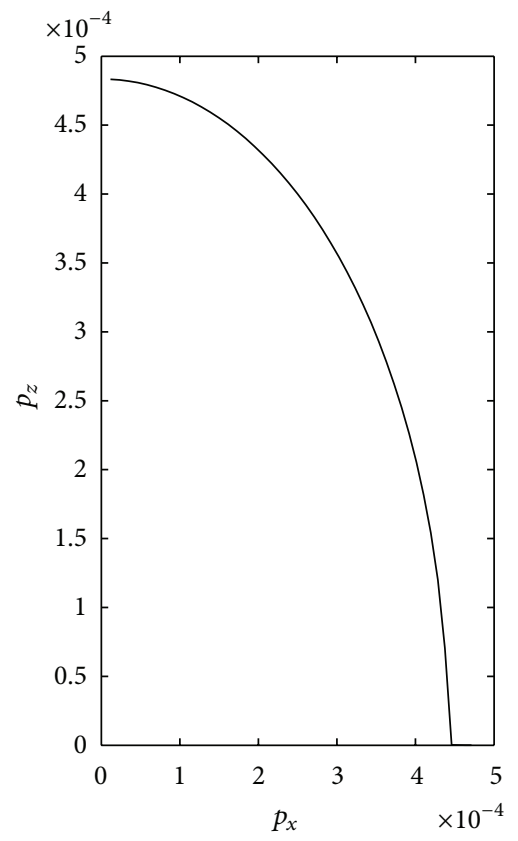

(c)

FIGURE 3: Variation of vertical component of slowness with horizontal component of slowness; (a) $q P_{1}$ wave, (b) $q S_{1}$ wave, and (c) $q P_{2}$ wave.

the slowness associated with the $q P_{1}$ wave is minimum while that associated with the $q P_{2}$ wave is maximum, which verifies the fact that the velocity of $q P_{1}$ wave is maximum while that of the $q P_{2}$ wave is minimum. The value of the vertical component of the slowness helps in deciding the nomenclature of the waves propagating in the medium. It is also clear from this figure that the first of all, $q P_{1}$, wave becomes nonpropagating.

Figure 4 displays the variation of real part of mechanical displacements and electric potentials with $x_{3}$. It is observed that mechanical displacements and electric potentials decrease with $x_{3}$ and become zero for large value of $x_{3}$. Thus the amplitudes of the waves decrease as they travel far away from the interface and die out after a very long distance.

\section{Conclusion}

In the present paper the wave interaction of piezoelastic waves at fluid-porous piezoelectric solid half-space is studied. The analytical expression of the Christoffel equationis 

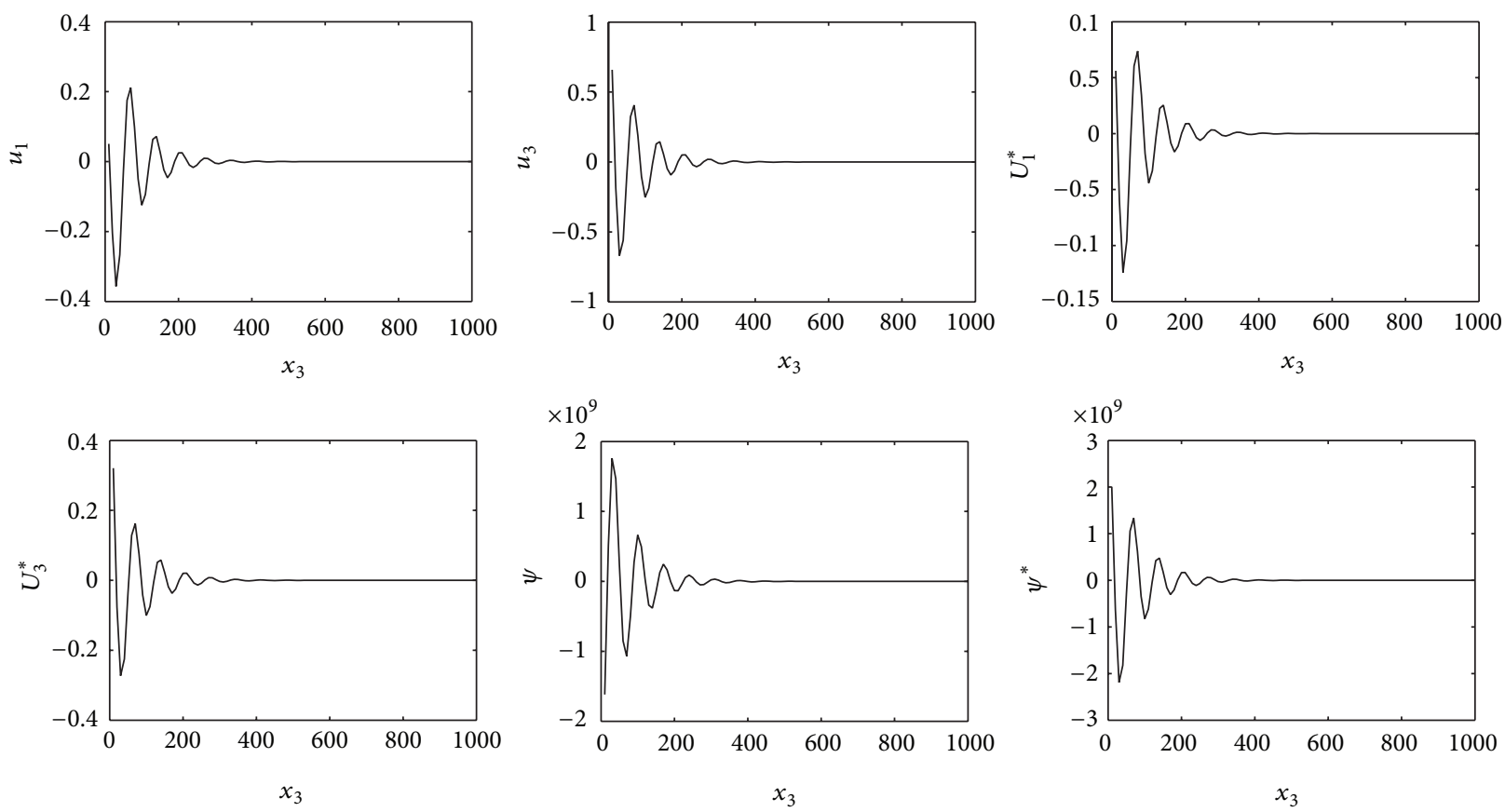

FIGURE 4: Variation of real part of mechanical displacements and electric potentials with $x_{3}$.

derived. The vertical slowness of the five coupled waves propagating in a medium is obtained from the solution of Christoffel equation. The analytical solution of the mechanical displacements, electric displacements, electric potentials, and stress in the porous piezoelectric half-space and fluid half-space is formulated. The behavior of angle of refraction, amplitude ratios, displacements, potentials, and vertical component of slowness relative to various parameters is studied numerically for particular model. It is found that major portion of incident energy gets reflected back in case of studied model which signifies the fact that the transmitting medium is much denser. The displacements and potentials decrease with the increase in $x_{3}$ and become zero for large value of $x_{3}$.

\section{Appendix}

Consider

$$
\begin{aligned}
c_{1}= & \beta_{1} z_{1}-\beta_{5} z_{10}+\beta_{9} z_{17}-\beta_{13} z_{26}, \\
c_{2}= & \beta_{2} z_{1}+\beta_{1} z_{2}-\beta_{6} z_{10}-\beta_{5} z_{11}+\beta_{10} z_{17} \\
& +\beta_{9} z_{18}-\beta_{14} z_{26}-\beta_{13} z_{27}, \\
c_{3}= & \beta_{3} z_{1}+\beta_{2} z_{2}+\beta_{1} z_{3}-\beta_{7} z_{10}-\beta_{6} z_{11}+\beta_{11} z_{17} \\
& +\beta_{10} z_{18}+\beta_{9} z_{19}-\beta_{15} z_{26}-\beta_{14} z_{27}-\beta_{13} z_{28}, \\
c_{4}= & \beta_{4} z_{1}+\beta_{3} z_{2}+\beta_{2} z_{3}-\beta_{8} z_{10}-\beta_{7} z_{11}+\beta_{12} z_{17} \\
& +\beta_{11} z_{18}+\beta_{10} z_{19}-\beta_{16} z_{26}-\beta_{15} z_{27}-\beta_{14} z_{28},
\end{aligned}
$$

$$
\begin{aligned}
& c_{5}= \beta_{4} z_{2}+\beta_{3} z_{3}+\beta_{12} z_{18}+\beta_{11} z_{19}-\beta_{8} z_{11} \\
&-\beta_{16} z_{27}-\beta_{15} z_{28} ; \\
& c_{6}= \beta_{4} z_{3}+\beta_{12} z_{19}-\beta_{16} z_{28} \\
& \beta_{1}= q_{1} z_{12}-q_{4} z_{14}+q_{7} z_{15} ; \\
& \beta_{2}= q_{2} z_{12}+q_{1} z_{13}-q_{5} z_{14}+q_{8} z_{15}+q_{7} z_{16} ; \\
& \beta_{3}=q_{3} z_{12}+q_{2} z_{13}-q_{6} z_{14}+q_{9} z_{15}+q_{8} z_{16} ; \\
& \beta_{4}=q_{3} z_{13}+q_{9} z_{16} ; \\
& \beta_{5}=q_{1} z_{4}-q_{4} z_{6}+q_{7} z_{8} ; \\
& \beta_{6}=q_{2} z_{4}+q_{1} z_{5}-q_{5} z_{6}-q_{4} z_{7}+q_{8} z_{8}+q_{7} z_{9} ; \\
& \beta_{7}=q_{3} z_{4}+q_{2} z_{5}-q_{6} z_{6}-q_{5} z_{7}+q_{9} z_{8}+q_{8} z_{9} ; \\
& \beta_{8}=q_{3} z_{5}-q_{6} z_{7}+q_{9} z_{9} ; \\
& \beta_{9}=r_{1} z_{4}-r_{4} z_{6}+r_{7} z_{8} ; \\
& \beta_{10}=r_{2} z_{4}+r_{1} z_{5}-r_{5} z_{6}-r_{4} z_{7}+r_{8} z_{8}+r_{7} z_{9} ; \\
& \beta_{11=}=r_{3} z_{4}+r_{2} z_{5}-r_{6} z_{6}-r_{5} z_{7}+r_{9} z_{8}+r_{8} z_{9} ; \\
& \beta_{12}=r_{3} z_{5}-r_{6} z_{7}+r_{9} z_{9} ; \\
& \beta_{13}=s_{1} z_{4}-s_{4} z_{6}+s_{7} z_{8} ; \\
& \beta_{14}=s_{2} z_{4}+s_{1} z_{5}-s_{5} z_{6}-s_{4} z_{7}+s_{8} z_{8}+s_{7} z_{9} ; \\
& \beta_{15}=s_{3} z_{4}+s_{2} z_{5}-s_{6} z_{6}-s_{5} z_{7}+s_{9} z_{8}+s_{8} z_{9} ; \\
& \beta_{16}=s_{3} z_{5}-s_{6} z_{7}+s_{9} z_{9} ;
\end{aligned}
$$




$$
\begin{aligned}
& q_{1}=z_{22} z_{33}-z_{24} z_{31} \\
& q_{2}=z_{22} z_{34}+z_{23} z_{33}-z_{25} z_{31}-z_{24} z_{32} \text {; } \\
& q_{3}=z_{23} z_{34}-z_{25} z_{32} \\
& q_{4}=z_{20} z_{33}-z_{24} z_{29} \text {; } \\
& q_{5}=z_{20} z_{34}+z_{21} z_{33}-z_{24} z_{30}-z_{25} z_{29} ; \\
& q_{6}=z_{21} z_{34}-z_{25} z_{30} ; \quad q_{7}=z_{20} z_{31}-z_{22} z_{29} ; \\
& q_{8}=z_{20} z_{32}+z_{21} z_{31}-z_{23} z_{29}-z_{22} z_{30} \text {; } \\
& q_{9}=z_{21} z_{32}-z_{23} z_{30} \text {; } \\
& r_{1}=z_{14} z_{33}-z_{15} z_{31} \\
& r_{2}=z_{14} z_{34}-z_{16} z_{31}-z_{15} z_{32} ; \\
& r_{3}=-z_{16} z_{32} ; \quad r_{4}=z_{12} z_{33}-z_{15} z_{29} ; \\
& r_{5}=z_{12} z_{34}+z_{13} z_{33}-z_{16} z_{29}-z_{15} z_{30} \text {; } \\
& r_{6}=z_{13} z_{34}-z_{16} z_{30} ; \quad r_{7}=z_{12} z_{31}-z_{14} z_{29} ; \\
& r_{8}=z_{12} z_{32}-z_{14} z_{30}+z_{13} z_{31} ; \quad r_{9}=z_{13} z_{32} ; \\
& s_{1}=z_{14} z_{24}-z_{15} z_{22} ; \\
& s_{2}=z_{14} z_{25}-z_{16} z_{22}-z_{15} z_{23} ; \\
& s_{3}=-z_{16} z_{23} ; \quad s_{4}=z_{12} z_{24}-z_{15} z_{20} ; \\
& s_{5}=z_{12} z_{25}+z_{13} z_{24}-z_{16} z_{20}-z_{15} z_{21} \text {; } \\
& s_{6}=z_{13} z_{25}-z_{16} z_{21} ; \quad s_{7}=z_{12} z_{22}-z_{14} z_{20} ; \\
& s_{8}=z_{12} z_{23}-z_{14} z_{21}+z_{13} z_{22} ; \quad s_{9}=z_{13} z_{23} ; \\
& z_{1}=e_{33} y_{1}+\zeta_{33} y_{6} \\
& z_{2}=\left(e_{15} y_{1}+\zeta_{15} y_{6}\right) p_{x}^{2}+\left(c_{44}+c_{13}\right) p_{x} \\
& +e_{33} y_{2}+\zeta_{33} y_{7} \\
& z_{3}=\left(e_{15} y_{2}+\zeta_{15} y_{7}\right) p_{x}^{2} ; \quad z_{4}=e_{33} y_{3}+\zeta_{33} y_{8}+c_{33} ; \\
& z_{5}=\left(e_{15} y_{3}+\zeta_{15} y_{8}+c_{44}\right) p_{x}^{2}-\rho_{11} \text {; } \\
& z_{6}=e_{33} y_{4}+\zeta_{33} y_{9}+m_{33} p_{x} \\
& z_{7}=\left(e_{15} y_{4}+\zeta_{15} y_{9}\right) p_{x}^{2} \\
& z_{8}=e_{33} y_{5}+\zeta_{33} y_{10}+m_{33} \text {; } \\
& z_{9}=\left(e_{15} y_{5}+\zeta_{15} y_{10}\right) p_{x}^{2}-\rho_{12} ; \\
& z_{10}=\widetilde{\zeta}_{3} y_{1}+e_{3}^{*} y_{6} ; \quad z_{11}=m_{11} p_{x}+\widetilde{\zeta}_{3} y_{2}+e_{3}^{*} y_{7} ; \\
& z_{12}=m_{33}+\widetilde{\zeta}_{3} y_{3}+e_{3}^{*} y_{8} \\
& z_{13}=-\rho_{12} ; \quad z_{14}=R p_{x}+\widetilde{\zeta}_{3} y_{4}+e_{3}^{*} y_{9} ; \\
& z_{15}=R+\widetilde{\zeta}_{3} y_{5}+e_{3}^{*} y_{10} ; \\
& z_{16}=-\rho_{22} ; \quad z_{17}=-y_{1} \xi_{33}-y_{6} A_{33} \text {; } \\
& z_{18}=\left(-y_{1} \xi_{11}-y_{6} A_{11}\right) p_{x}^{2}+\left(e_{15}+e_{31}\right) p_{x} \\
& -\xi_{33} y_{2}-A_{33} y_{7} \text {; } \\
& z_{19}=-\left(y_{2} \xi_{11}+y_{7} A_{11}\right) p_{x}^{2} ; \\
& z_{20}=e_{33}-y_{3} \xi_{33}-y_{8} A_{33} \text {; } \\
& z_{21}=\left(e_{15}-y_{3} \xi_{11}-y_{8} A_{11}\right) p_{x}^{2} \text {; } \\
& z_{22}=\widetilde{\zeta}_{3} p_{x}-A_{33} y_{9}-\xi_{33} y_{4} ; \\
& z_{23}=-\left(y_{4} \xi_{11}+y_{9} A_{11}\right) p_{x}^{2} \text {; } \\
& z_{24}=-y_{10} A_{33}-y_{5} \xi_{33}+\widetilde{\zeta}_{3} ; \\
& z_{25}=-\left(y_{5} \xi_{11}+y_{10} A_{11}\right) p_{x}^{2} ; \\
& z_{26}=-y_{1} A_{33}-y_{6} \xi_{33}^{*} ; \\
& z_{27}=\left(-y_{1} A_{11}-y_{6} \xi_{11}^{*}\right) p_{x}^{2}+\left(\zeta_{15}+\zeta_{31}\right) p_{x} \\
& -y_{2} A_{33}-y_{7} \xi_{33}^{*} \text {; } \\
& z_{28}=-\left(y_{2} A_{11}+y_{7} \xi_{11}^{*}\right) p_{x}^{2} \text {; } \\
& z_{29}=\zeta_{33}-y_{3} A_{33}-y_{8} \xi_{33}^{*} \text {; } \\
& z_{30}=\left(\zeta_{15}-y_{3} A_{11}-y_{8} \xi_{11}^{*}\right) p_{x}^{2} ; \\
& z_{31}=e_{3}^{*} p_{x}-\xi_{33}^{*} y_{9}-A_{33} y_{4} \text {; } \\
& z_{32}=-\left(y_{4} A_{11}+y_{9} \xi_{11}^{*}\right) p_{x}^{2} ; \\
& z_{33}=-y_{10} \xi_{33}^{*}-y_{5} A_{33}+e_{3}^{*} \text {; } \\
& z_{34}=-\left(y_{5} A_{11}+y_{10} \xi_{11}^{*}\right) p_{x}^{2} ; \\
& y_{1}=\frac{-c_{44} d}{p_{x}} \\
& y_{2}=-\frac{\left(c_{11} p_{x}^{2}-\rho_{11}\right) d}{p_{x}}-\frac{\left(m_{11} p_{x}^{2}-\rho_{12}\right)}{p_{x} \widetilde{\zeta}_{3}} \\
& +\frac{\left(m_{11} p_{x}^{2}-\rho_{12}\right)\left(e_{15}+e_{31}\right) d}{p_{x} \widetilde{\zeta}_{3}} \\
& y_{3}=\frac{\left(m_{33}\left(e_{15}+e_{31}\right) d-m_{33}\right)}{\widetilde{\zeta}_{3}}-\left(c_{13}+c_{44}\right) d ; \\
& y_{4}=-\frac{\left(m_{11} p_{x}^{2}-\rho_{12}\right) d}{p_{x}}-\frac{\left(R p_{x}^{2}-\rho_{22}\right)}{p_{x} \widetilde{\zeta}_{3}} \\
& +\frac{\left(R p_{x}^{2}-\rho_{22}\right)\left(e_{15}+e_{31}\right) d}{p_{x} \widetilde{\zeta}_{3}} \\
& y_{5}=\frac{\left(R\left(e_{15}+e_{31}\right) d-R\right)}{\widetilde{\zeta}_{3}}-m_{11} d ;
\end{aligned}
$$




$$
\begin{aligned}
& y_{6}=\frac{c_{44} \widetilde{\zeta}_{3} d}{p_{x} e_{3}^{*}} ; \\
& y_{7}=\frac{\left(c_{11} p_{x}^{2}-\rho_{11}\right) d \widetilde{\zeta}_{3}}{p_{x} e_{3}^{*}}-\frac{\left(m_{11} p_{x}^{2}-\rho_{12}\right)\left(e_{15}+e_{31}\right) d}{p_{x} e_{3}^{*}} ; \\
& y_{8}=\frac{\left(c_{13}+c_{44}\right) d \widetilde{\zeta}_{3}}{e_{3}^{*}}-\frac{m_{33}\left(e_{15}+e_{31}\right) d}{e_{3}^{*}} ; \\
& y_{9}=\frac{\left(m_{11} p_{x}^{2}-\rho_{12}\right) d \widetilde{\zeta}_{3}}{p_{x} e_{3}^{*}}-\frac{\left(R p_{x}^{2}-\rho_{22}\right)\left(e_{15}+e_{31}\right) d}{p_{x} e_{3}^{*}} ; \\
& y_{10}=\frac{m_{11} d \widetilde{\zeta}_{3}}{e_{3}^{*}-\frac{R\left(e_{15}+e_{31}\right) d}{e_{3}^{*}} ;} \\
& d=\frac{e_{3}^{*}}{\left(e_{3}^{*}\left(e_{15}+e_{31}\right)-\widetilde{\zeta}_{3}\left(\zeta_{15}+\zeta_{31}\right)\right)} .
\end{aligned}
$$

\section{Conflict of Interests}

There is no conflict of interests regarding the publication of this paper.

\section{References}

[1] B. Noorbehesht and G. Wade, "Spatial frequency characteristics of opto-acoustic transducers," Acoustical Imaging: Proceedings of the International Symposium, vol. 9, pp. 139-154, 1980.

[2] B. A. Auld, Acoustic Fields and Waves in Solids, John Wiley and Sons, New York, NY, USA, 1973.

[3] E. Dieulesaint and D. Royer, Elastic Waves in Solids: Applications To Signal Processing, Wiley, New York, NY, USA, 1980.

[4] V. Z. Parton and B. A. Kudryavtsev, Electromagnetoelasticity: Piezoelectrics and Electrically Conductive Solids, Gordon and Beach, New York, NY, USA, 1988.

[5] B. Noorbehesht and G. Wade, "Reflection and transmission of plane elastic waves at the boundary between piezoelectric materials and water," Journal of the Acoustical Society of America, vol. 67, no. 6, pp. 1947-1953, 1980.

[6] B. A. Auld, "Wave propagation and resonance in piezoelectric materials," Journal of the Acoustical Society of America, vol. 70, no. 6, pp. 1577-1585, 1981.

[7] V. I. Alshits, J. Lothe, and V. N. Lyubimov, "The phase shift for reflection of elastic waves in hexagonal piezoelectric crystals," Wave Motion, vol. 6, no. 3, pp. 259-264, 1984.

[8] A. H. Nayfeh and H.-T. Chien, "Wave propagation interaction with free and fluid-loaded piezoelectric substrates," Journal of the Acoustical Society of America, vol. 91, no. 6, pp. 3126-3135, 1992.

[9] A. G. Every and V. I. Neiman, "Reflection of electroacoustic waves in piezoelectric solids: mode conversion into four bulk waves," Journal of Applied Physics, vol. 71, no. 12, pp. 6018-6024, 1992.

[10] L. P. Zinchuk and A. N. Podlipenets, "Propagation of shear waves in piezoelectric "superlattice- substrate" structures," Journal of Mathematical Sciences, vol. 101, no. 6, pp. 3688-3693, 2000 .
[11] L. P. Zinchuk and A. N. Podlipenets, "Dispersion equations for rayleigh waves in a piezoelectric periodically layered structure," Journal of Mathematical Sciences, vol. 103, no. 3, pp. 398-403, 2001.

[12] B. D. Zaitsev and I. E. Kuznetsova, "The energy density and power flow of acoustic waves propagating in piezoelectric materials," IEEE Transactions on Ultrasonics, Ferroelectrics, and Frequency Control, vol. 50, no. 12, pp. 1762-1765, 2003.

[13] E. Le Clezio and A. Shuvalov, "Transmission of acoustic waves through piezoelectric plates: modeling and experiment," in IEEE Ultrasonics Symposium, pp. 553-556, August 2004.

[14] S. I. Burkov, B. P. Sorokin, D. A. Glushkov, and K. S. Aleksandrov, "Theory and computer simulation of the reflection and refraction of bulk acoustic waves in piezoelectrics under the action of an external electric field," Crystallography Reports, vol. 50, no. 6, pp. 986-993, 2005.

[15] S. I. Burkov, B. P. Sorokin, K. S. Aleksandrov, and A. A. Karpovich, "Reflection and refraction of bulk acoustic waves in piezoelectrics under uniaxial stress," Acoustical Physics, vol. 55, no. 2, pp. 178-185, 2009.

[16] A. N. Darinskii, E. Le Clezio, and G. Feuillard, "The role of electromagnetic waves in the reflection of acoustic waves in piezoelectric crystals," Wave Motion, vol. 45, no. 4, pp. 428-444, 2008.

[17] A.-E. N. Abd-Alla and F. A. Alsheikh, "Reflection and refraction of plane quasi-longitudinal waves at an interface of two piezoelectric media under initial stresses," Archive of Applied Mechanics, vol. 79, no. 9, pp. 843-857, 2009.

[18] K. Y. Hashimoto and M. Yamaguchi, "Elastic, piezoelectric and dielectric properties of composite materials," Proceeding IEEE Ultrasonics Symposium, vol. 2, pp. 697-702, 1986.

[19] H. Banno, “Theoretical equations for dielectric, piezoelectric and elastic properties of flexible composite consisting of polymer and ceramic powder of two different materials," Ferroelectric, vol. 95, no. 1, pp. 111-115, 1989.

[20] W. A. Smith and B. A. Auld, "Modeling 1-3 composite piezoelectrics: thickness-mode oscillations," IEEE Transactions on Ultrasonics, Ferroelectrics, and Frequency Control, vol. 38, no. 1, pp. 40-47, 1991.

[21] H. Banno, "Effects of porosity on dielectric, elastic and electromechanical properties of $\mathrm{Pb}(\mathrm{Zr}, \mathrm{Ti}) \mathrm{O}_{3}$ ceramics with open pores: a theoretical approach," Japanese Journal of Applied Physics: Regular Papers and Short Notes and Review Papers, vol. 32, no. 9, pp. 4214-4217, 1993.

[22] C. R. Bowen, A. Perry, A. C. F. Lewis, and H. Kara, "Processing and properties of porous piezoelectric materials with high hydrostatic figures of merit," Journal of the European Ceramic Society, vol. 24, no. 2, pp. 541-545, 2004.

[23] B. Praveenkumar, H. H. Kumar, and D. K. Kharat, "Characterization and microstructure of porous lead zirconate titanate ceramics," Bulletin of Materials Science, vol. 28, no. 5, pp. 453455, 2005.

[24] D. Piazza, L. Stoleriu, L. Mitoseriu, A. Stancu, and C. Galassi, "Characterisation of porous PZT ceramics by first-order reversal curves (FORC) diagrams," Journal of the European Ceramic Society, vol. 26, no. 14, pp. 2959-2962, 2006.

[25] H. L. Zhang, J.-F. Li, and B.-P. Zhang, "Microstructure and electrical properties of porous PZT ceramics derived from different pore-forming agents," Acta Materialia, vol. 55, no. 1, pp. 171-181, 2007. 
[26] A. K. Vashishth and V. Gupta, "Vibrations of porous piezoelectric ceramic plates," Journal of Sound and Vibration, vol. 325, no. 4-5, pp. 781-797, 2009.

[27] A. K. Vashishth and V. Gupta, "Wave propagation in transversely isotropic porous piezoelectric materials," International Journal of Solids and Structures, vol. 46, no. 20, pp. 3620-3632, 2009.

[28] A. K. Vashishth and V. Gupta, "Uniqueness theorem, theorem of reciprocity, and eigenvalue problems in linear theory of porous piezoelectricity," Applied Mathematics and Mechanics, vol. 32, no. 4, pp. 479-494, 2011.

[29] A. K. Vashishth and V. Gupta, "Reflection and transmission phenomena in poroelastic plate sandwiched between fluid half space and porous piezoelectric half space," Smart Materials Research, vol. 2013, Article ID 767019, 8 pages, 2013.

[30] R. Kar-Gupta and T. A. Venkatesh, "Electromechanical response of porous piezoelectric materials," Acta Materialia, vol. 54, no. 15, pp. 4063-4078, 2006. 

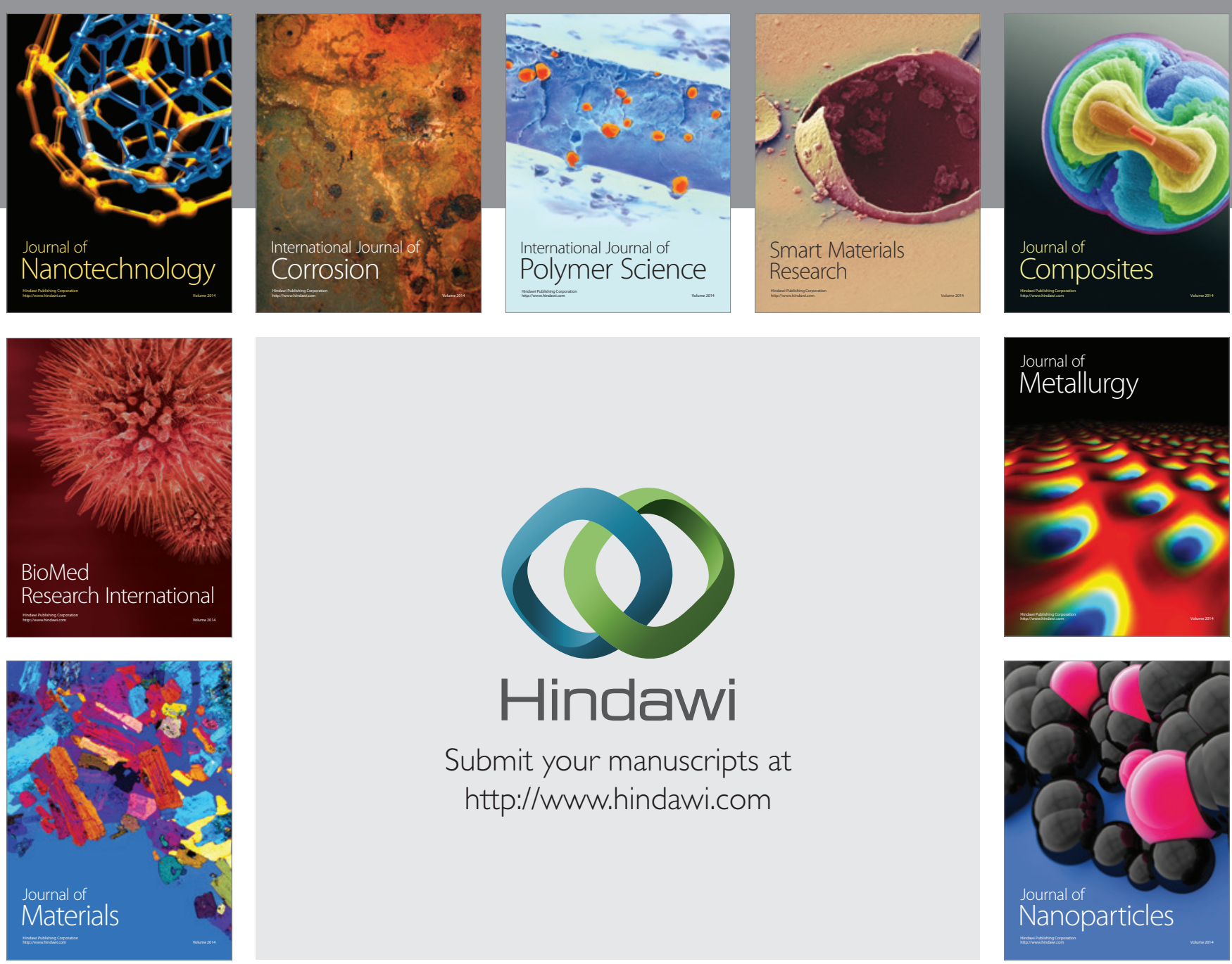

Submit your manuscripts at http://www.hindawi.com
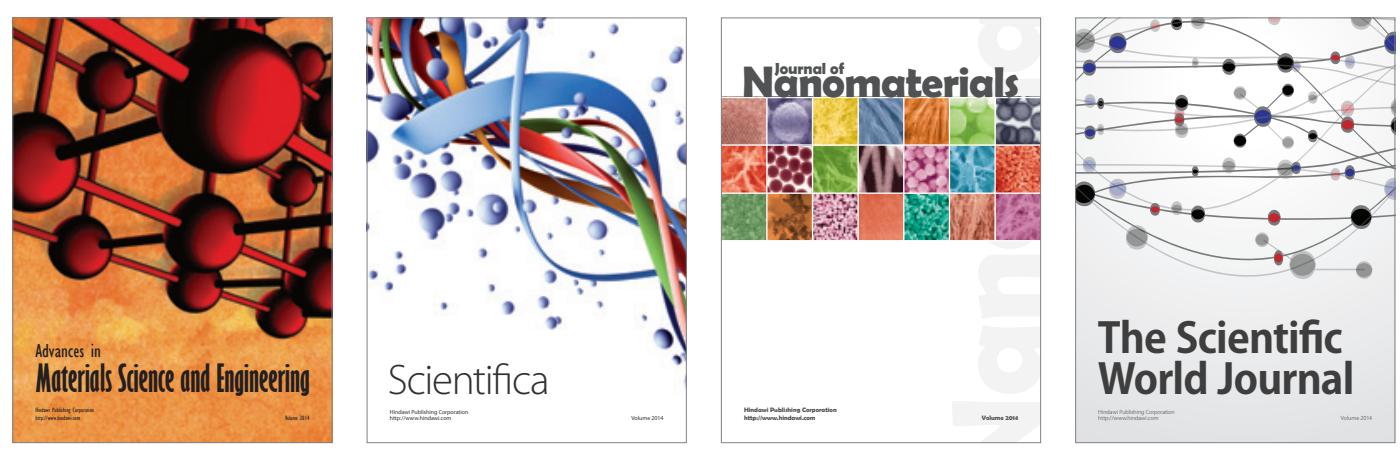

\section{The Scientific World Journal}
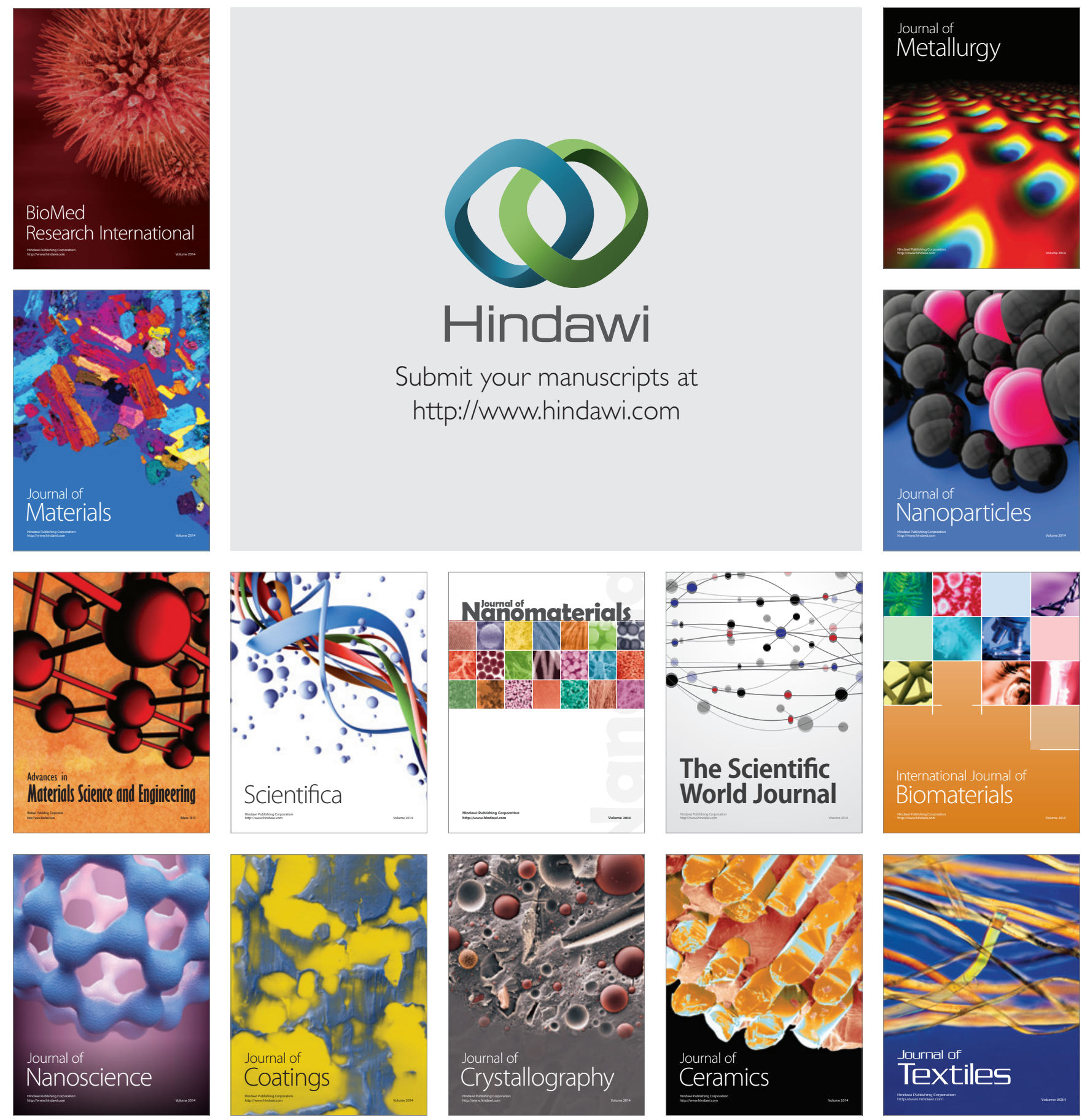